\title{
Ra Ximhai
}

Revista de Sociedad, Cultura y Desarrollo Sustentable

Ra Ximhai

Universidad Autónoma Indígena de México

ISSN: 1665-0441

México

ESTIMULACIÓN TEMPRANA DEL CRECIMIENTO DEL EPICOTILO EN PLÁNTULAS

DE Pinus montezumae LAMB

Nicolás Calderón Paniagua, Jesús Jasso Mata, José de Jesús Martínez Hernández, Jesús Vargas Hernández y Adrián Gómez González

Ra Ximhai, septiembre-diciembre, año/Vol.2, Número 3

Universidad Autónoma Indígena de México

Mochicahui, El Fuerte, Sinaloa. pp. 847-864

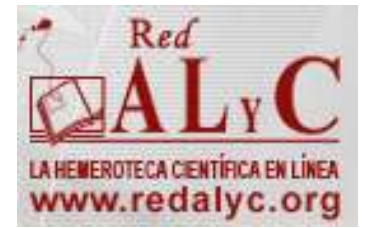




\title{
ESTIMULACIÓN TEMPRANA DEL CRECIMIENTO DEL EPICOTILO EN PLÁNTULAS \\ DE Pinus montezumae LAMB
}

\section{EARLY GROWTH STIMULATION OF THE EPICOTILO IN SEEDLING OF Pinus montezumae LAMB}

\author{
Nicolás Calderón-Paniagua'; Jesús Jasso-Mata ${ }^{1}$; José de Jesús Martínez-Hernández ${ }^{2}$; \\ Jesús Vargas-Hernández ${ }^{1}$ y Adrián Gómez-González ${ }^{2}$ \\ Programa Forestal Colegio de Posgraduados. Correo electrónico: jejama@colpos.mx y vargashj@colpos.mx. ${ }^{2}$ Programa Botánica. \\ Colegio de Postgraduados.
}

\section{RESUMEN}

Pinus montezumae Lamb., se considera una especie maderable de gran importancia económica, de la que se obtienen un gran número de derivados. Presenta una amplia distribución en México y se ha utilizado en la recuperación de suelos degradados; además de que se recomienda para plantaciones ornamentales (Eguiluz, 1978). Sin embargo, poco se utiliza en plantaciones forestales comerciales ya que durante la etapa de vivero, presenta un nulo crecimiento en altura. Con el propósito de acelerar las tasas de crecimiento inicial en Pinus montezumae Lamb., se cultivaron cuatro familias de esta especie (056, 066, 074 y 083) en hidroponia, aplicándose dos soluciones nutritivas (Cooper y Estándar) y utilizando un diseño experimental en bloques al azar. Las plántulas se trasplantaron a un sistema hidropónico semiautomático, 40 días después de germinadas con un diámetro y una altura promedio de $1 \mathrm{~mm}$ y $5 \mathrm{~cm}$ respectivamente. La duración del experimento fue de 30 semanas ( 7 meses) tiempo en el cual se hicieron tres muestreos destructivos con el objetivo de medir el crecimiento en longitud y biomasa, en forma dinámica. Las plántulas de la familia 056 fueron significativamente superiores a las de la familia 066, al alcanzar en las etapas finales del experimento las mayores magnitudes en el diámetro del hipocotilo, longitud del epicotilo y materia seca de las plantas; entre soluciones nutritivas se detectó una superioridad de la solución nutritiva Cooper con respecto a la solución nutritiva Estándar, al desarrollar las plantas nutridas con la primera solución mayores dimensiones en las variables medidas principalmente durante la etapa final del experimento.

Palabras clave: Pinus montezumae Lamb., solución nutritiva, familia, hidroponia, crecimiento del epicotilo.

\section{SUMMARY}

Pinus montezumae Lamb, is considered timber-yielding specie of great economic importance, a great number of products are obtained from it. It is widely distributed in Mexico and it has been used to recover eroded soils; it is also recommended for ornamental plantations (Eguiluz, 1978). However, no commercial forest plantations are established because of the early slow growth height. In order to study the initial growth rates in Pinus montezumae Lamb, four families of this specie (056, 066, 074 y 083) were grown in hydroponics, two nutritive solutions (Cooper and Standard) were applied in a randomized block design. 40 days germinated seedlings were transplanted to a hydroponic and semiautomatic system. The seedlings averaged $1 \mathrm{~mm}$ in diameter and $5 \mathrm{~cm}$ in height. The experiment lasted a period of 30 weeks ( 7 months). Three destructive samples were applied to measure seedling height, growth and biomass seedlings. Comparison among seedlings indicated that family 056 was significantly better than the family 066 , at the end of the experiment. Comparison results of all variables indicated that the Cooper solution was superior to the Standard one at the end of the study.

Key words: Pinus montezumae Lamb, nutritive solution, family, hidroponics, epicotyl growth. 


\section{INTRODUCCIÓN}

Pinus montezumae Lamb., se considera una especie maderable de gran importancia económica, se utiliza en la fabricación de triplay, celulosa, papel, cajas de empaque, puntales para mina, postes para cableados, ebanistería, duela y en la industria constructora, además de ser una especie resinera importante. La especie se ha utilizado en la recuperación de suelos degradados y se recomienda para plantaciones ornamentales (Eguiluz, 1978).

El crecimiento inicial de la especie se caracteriza por una nula elongación del epicotilo y una abundante producción de hojas primarias y secundarias (estado cespitoso) durante un periodo que puede durar de 2 a 6 años (Mirov, 1967), lo que resulta un problema serio tanto en vivero porque la planta requiere una mayor permanencia en el mismo, como en el bosque en forma natural, ya que a medida que el estado cespitoso se prolonga las plántulas quedan expuestas a la competencia ecológica originada por hierbas anuales y especies leñosas de rápido crecimiento (Becerra, 1990).

Sin embargo, por la gran importancia económica y ecológica de la especie resulta necesario realizar investigaciones enfocadas a la aplicación de técnicas genéticas o ambientales que ayuden a reducir el tiempo que tarda la especie en estado cespitoso, para que de esta manera sea más atractiva no sólo en estado adulto, sino también como plántula para utilizarse en reforestación y plantaciones comerciales.

Varias investigaciones han sido conducidas con el objetivo de observar el efecto de la intensidad de la luz solar en el crecimiento inicial de Pinus montezumae Lamb. Vela y Hernández (1968), Musálem (1985) y Vera (1986). Sin embargo, los resultados no han sido satisfactorios, ya que no se ha encontrado la elongación temprana del epicotilo.

Allen (1958) citado por Hare (1984) logró estimular el crecimiento temprano en altura en Pinus palustris con ácido giberélico. Por su parte, Kossuth (1981) reporta que el estimulo 
aumenta al aplicar a las plantas ácido giberélico en combinación con benciladenina en una relación de $3: 1$; respectivamente.

En Pinus palustris Mill., se ha logrado romper el estado cespitoso, en algunos casos, con la aplicación de fertilizantes tanto en plantas en vivero como en sitios de regeneración (Hughes et al., 1971). Así mismo, Hinsley (1980) y Lewis et al., (1985) obtuvieron resultados semejantes al aplicar soluciones nutritivas compuestas con nitrógeno, fósforo, potasio, calcio y magnesio en plántulas de Pinus palustris de dos años de edad.

Los objetivos planteados en el presente trabajo fueron: 1) Cuantificar el crecimiento en longitud y biomasa de plántulas de diferentes familias de Pinus montezumae Lamb., cultivadas en un sistema hidropónico; 2) Evaluar el efecto de dos soluciones nutritivas (Cooper y Estándar) sobre el crecimiento y desarrollo de plántulas de Pinus montezumae Lamb., en un periodo de 1.5 a 8.5 meses después del trasplante.

\section{MATERIALES Y MÉTODOS}

El experimento se estableció en un invernadero del Campus San Luis Potosí, del Colegio de Postgraduados, ubicado en Salinas de Hgo. SLP., a una altitud de 2070 msnm y localizado en las siguientes coordenadas geográficas: $22^{\circ} 37^{\prime}$ de latitud norte y $101^{\circ} 42^{\prime}$ de longitud oeste (Cortés, 1987).

El microambiente en el que se desarrollaron las plantas fue el siguiente: durante los meses de diciembre a marzo las temperaturas mínimas promedio mensuales registradas estuvieron entre 0 y $10^{\circ} \mathrm{C}$ y las máximas entre 30 y $35^{\circ} \mathrm{C}$, y en el periodo de abril a junio las temperaturas mínimas promedio oscilaron entre 10 y $15^{\circ} \mathrm{C}$ y las máximas promedio entre 35 y $40^{\circ} \mathrm{C}$. Durante los días soleados, se pusieron a funcionar los extractores de aire caliente del invernadero, además se agregó agua al muro húmedo. En este segundo periodo se presentaron las mayores velocidades de crecimiento de las plántulas de Pinus montezumae Lamb.

El microambiente del sistema radical de las plantas fue diferente entre soluciones nutritivas, el pH de la SNCOO varió en promedio entre 5.5 y 6.5 y la conductividad eléctrica 
promedio durante el experimento fue de 1.8; en la SNEST el pH estuvo entre 5.5 y 6.7 y la CE promedio durante todo el experimento fue de 1.7.

Como material vegetal se utilizaron plántulas de cuatro familias de Pinus montezumae Lamb., (056, 066, 074 y 066) germinadas en charolas de plástico, utilizando como sustrato arena de río. La semilla fue colectada en el "Área Semillera” del Campo Experimental Forestal San Juan Tetla, Puebla. Las plántulas se trasplantaron a un sistema hidropónico semiautomático, 40 días después de germinadas con un diámetro y una altura promedio de $1 \mathrm{~mm}$ y $5 \mathrm{~cm}$ respectivamente.

Como sustrato se utilizó tezontle rojo, tamizado a una granulometría entre 2 y $4 \mathrm{~mm}$. y desinfectado con una solución de hipoclorito de sodio (Clorales con 6\% de $\mathrm{Cl}$ activo) diluida al $0.2 \%$. Después se lavó con agua de la llave y se puso a secar al aire libre por 48 horas. Se utilizaron macetas de PVC de una capacidad de $2.3 \mathrm{~L}$.

El experimento consistió de un arreglo factorial 2x4 con un diseño experimental en bloques al azar, con dos soluciones nutritivas (Cooper y Estándar), cuatro familias de Pinus montezumae Lamb., (056, 066, 074 y 083) y cuatro repeticiones (bloques) con tres plantas de cada familia por bloque.

Se proporcionó agua y nutrimentos a la planta con ayuda de mangueras tipo espagueti colocadas cerca del cuello de la raíz de cada planta y conectadas a un tubo distribuidor de la solución nutritiva. Al inicio del experimento se proporcionaron cuatro riegos diarios con agua destilada durante cuatro días para homogeneizar las condiciones de humedad y nutrición. Después se aplicaron las soluciones nutritivas diluidas al 10\%, elevando gradualmente sus concentraciones durante un mes, hasta alcanzar el $100 \%$ de las formulaciones de las SNEST y SNCOO respectivamente (Cuadro 1). 
Cuadro 1. Composición química de las soluciones nutritivas utilizadas en el experimento (ppm) (Furlani, 1995 y Calderón, 1995).

\begin{tabular}{cccccccccccc}
\hline & N & P & K & Ca & Mg & Fe & Mn & Cu & Zn & B & Mo \\
\hline SNCOO $^{1}$ & 200 & 60 & 300 & 170 & 50 & 12 & 2 & 0.1 & 0.1 & 0.3 & 0.3 \\
SNEST $^{2}$ & 150 & 40 & 225 & 210 & 40 & 12 & 2 & 0.1 & 0.2 & 0.6 & 0.05 \\
\hline
\end{tabular}

Durante la conducción del experimento se realizaron 14 cambios de solución nutritiva en función de la conductividad eléctrica de la misma, diariamente se midió y se ajustó a 5.5 el $\mathrm{pH}$ de ambas soluciones nutritivas utilizando un potenciómetro digital marca BECKMAN, modelo 3500 y adicionando $\mathrm{H}_{2} \mathrm{SO}_{4} 1 \mathrm{~N}$. La conductividad eléctrica de las soluciones nutritivas generalmente disminuyó al mismo tiempo que el volumen del contenedor. Sin embargo, ésta se controló aforando con una solución nutritiva preparada al $30 \%$ de su concentración normal. La conductividad eléctrica de las dos soluciones nutritivas utilizadas fue medida diariamente con un conductivímetro portátil marca HANNA instruments modelo HI 8733.

Las variables evaluadas fueron diámetro del hipocotilo (DH), longitud del epicotilo (LE), longitud de hojas primarias (LHP), número de hojas primarias (NHP), volumen de raíz fresca (VRF) y pesos fresco y seco de raíz, tallo, hojas y total. Para determinar el peso fresco y seco de cada uno de los órganos y de la planta completa se realizaron muestreos destructivos, cosechando la planta completa y separando en raíz, tallo y hojas, lavando las muestras y sometiéndolas a un proceso de secado en una estufa con circulación de aire a 70 ${ }^{\circ} \mathrm{C}$ durante 24 horas (Alcántar, 1992).

Se realizaron análisis de varianza para todas las variables evaluadas y cuando existió significancia estadística ( $\alpha=0.05)$, se aplicó una prueba de separación de medias (TUKEY). El paquete estadístico utilizado para realizar el análisis de la información fue el SAS (Statistical Analysis System) desarrollado por SAS Institute Inc. (1985). 


\section{RESULTADOS Y DISCUSIÓN}

\section{Caracteres morfológicos}

\section{Efecto de solución nutritiva}

Hubo diferencias estadísticas significativas a las 25 y 30 semanas después del trasplante (SDT), en las variables de diámetro del hipocotilo y longitud de epicotilo. En ambos casos la solución nutritiva "Cooper" presentó valores significativamente mayores a los registrados en las plántulas cultivadas en la solución nutritiva "Estándar" (Cuadro 2).

Las plántulas abastecidas con la solución nutritiva "Cooper" crecieron en promedio más en altura como consecuencia de alcanzar un mayor crecimiento del epicotilo, que las plántulas de Pinus montezumae Lamb. nutridas con la solución nutritiva "Estándar. En cambio, las variables de número de hojas primarias, longitud de hojas primarias y volumen de raíz fresca no presentaron diferencias significativas. Estas diferencias en el diámetro del hipocotilo y longitud de epicotilo encontradas entre soluciones nutritivas se le atribuyeron a la diferente concentración en macronutrimentos (NPK) entre ambas, (150, 40 y 225 ppm en la "Estándar" y 200, 60 y 300 ppm en la "Cooper". Lo anterior es coincidente con los resultados de Van Den Driesche (1982) quien encontró que el diámetro de algunas especies forestales se incrementa al aumentar la dosis de fertilización con nitrógeno. Similarmente, Mexal y Landis (1990), reportan que la nutrición con nitrógeno resulta un factor importante en el crecimiento del diámetro de plantas de coníferas a nivel vivero. Normalmente el crecimiento del diámetro del hipocotilo se le ha relacionado con el aumento de carbohidratos de reserva en plántulas de vivero (Thompson, 1984).

A diferencia de otros trabajos, donde se ha cultivado la especie con diferentes mezclas de sustratos (Vela y Hernández, 1966; Jasso, 1982 y 1990; Musálem, 1985; Vera, 1986 y Jiménez, 1998), en esta investigación la elongación del epicotilo, tuvo un comportamiento precoz, lo cual se le atribuyó a la hidroponia utilizada como medio de crecimiento. Al respecto, Ingestad, (1982) encontró que las tasas de crecimiento obtenidas por plántulas 
forestales cultivadas en hidroponia superaron por mucho, a las correspondientes tasas de crecimiento logradas cuando las plantas se cultivaron en diferentes mezclas de sustratos o suelos.

Cuadro 2. Características morfológicas de plántulas de Pinus montezumae Lamb., cultivadas en hidroponia.

\begin{tabular}{|c|c|c|c|c|}
\hline \multirow[t]{2}{*}{ Variable } & \multirow[t]{2}{*}{$\begin{array}{l}\text { Solución } \\
\text { nutritiva }\end{array}$} & \multicolumn{3}{|c|}{ Semanas después del trasplante } \\
\hline & & 20 & 25 & 30 \\
\hline Diámetro del & Cooper & $3.61 \mathbf{a}$ & $7.00 \mathbf{a}$ & $8.71 \mathbf{a}$ \\
\hline hipocotilo & Estándar & $3.67 \mathbf{a}$ & $5.58 \mathbf{b}$ & $7.54 \mathbf{b}$ \\
\hline $\operatorname{DMS}_{(\alpha=0.05)}$ & & 0.49 & 0.35 & 0.58 \\
\hline Número & Cooper & $98.06 \mathbf{a}$ & $142.50 \mathbf{a}$ & $189.06 \mathbf{a}$ \\
\hline hojas primarias & Estándar & 86.92 b & $132.56 \mathbf{a}$ & $184.37 \mathbf{a}$ \\
\hline $\operatorname{DMS}_{(\alpha=0.05)}$ & & 7.39 & 12.91 & 24.37 \\
\hline Longitud del & Cooper & $0.62 \mathbf{a}$ & $5.11 \mathbf{a}$ & $10.78 \mathbf{a}$ \\
\hline epicotilo & Estándar & $0.69 \mathbf{a}$ & $4.20 \mathbf{b}$ & $8.63 \mathbf{b}$ \\
\hline $\operatorname{DMS}_{(\alpha=0.05)}$ & & 0.34 & 0.58 & 0.84 \\
\hline Longitud de & Cooper & $13.69 \mathbf{a}$ & $13.94 \mathbf{a}$ & $12.06 \mathbf{a}$ \\
\hline hojas primarias & Estándar & $13.20 \mathrm{a}$ & $13.78 \mathbf{a}$ & $12.53 \mathbf{a}$ \\
\hline $\operatorname{DMS}_{(\alpha=0.05)}$ & & 1.45 & 1.42 & 0.73 \\
\hline Volumen de raíz & Cooper & $1.71 \mathrm{a}$ & $5.47 \mathbf{a}$ & $7.93 \mathbf{a}$ \\
\hline fresca & Estándar & $1.94 \mathbf{a}$ & $4.77 \mathbf{a}$ & $8.63 \mathrm{a}$ \\
\hline $\operatorname{DMS}_{(\alpha=0.05)}$ & & 0.80 & 1.47 & 1.80 \\
\hline
\end{tabular}

Los valores seguidos por la misma letra, no difieren estadísticamente (Tukey, 0.05).

En E.U.A se han obtenido resultados alentadores con Pinus palustris, una especie que presenta un estado cespitoso más acentuado que el Pinus montezumae Lamb., en la que se ha logrado romper el estado cespitoso con tratamientos a base de soluciones nutritivas compuestas con N, P, K, Ca y Mg y aplicadas a plantas de dos años de edad (Hinsley, 1980 y Lewis, et al., 1985).

El nitrógeno es el nutrimento más limitante en el crecimiento vegetativo, el cual consiste principalmente de la formación y crecimiento de nuevas hojas tallos y raíces. Debido a que los tejidos meristemáticos tienen un metabolismo muy activo de proteínas, los fotosintatos translocados a estos sitios se usan predominantemente en la síntesis de proteínas y ácidos 
nucleicos. Es por esta razón que durante el periodo de máximo crecimiento vegetativo de las plantas el $\mathrm{N}$ controla las tasas de crecimiento (Mengel y Kirkby, 1987).

En este estudio se considera al $\mathrm{N}$ como el nutrimento responsable de aumentar las tasas de crecimiento vegetativo, aún en la etapa de letargo del epicotilo. Otro nutrimento fundamental fue el $\mathrm{K}$ el cual actúa sinérgicamente con los reguladores de crecimiento (ácidos giberélico e indolacético) estimulando la elongación de tallos cortos (epicotilo) (Mengel y Kirkby, 1987).

\section{Efecto de familia}

Entre familias se detectaron diferencias significativas bien marcadas a las 25 y 30 semanas después del trasplante en todas las variables evaluadas. La familia 056 tuvo siempre un crecimiento mayor en todos los aspectos, lo que la mantuvo significativamente superior a la familia 066. Por otra parte las familias 074 y 083 mantuvieron un comportamiento intermedio en relación a las primeras. A las 30 semanas después del trasplante la familia 056 obtuvo un diámetro del hipocotilo de $9.92 \mathrm{~mm}$, que fue significativamente superior las familias 066, 074 y 083 que alcanzaron diámetros de 6.50, 8.32 y 7.75 mm, respectivamente (Cuadro 3).

El número de hojas primarias pudo haber estado asociado también con el crecimiento de las plántulas, se observó que la familia 056 con un número significativo mayor de hojas primarias a la familia 066 presentó una longitud de epicotilo mayor que ésta y por lo tanto mayor crecimiento en altura. Las plántulas de Pinus montezume Lamb. en este estudio en general no produjeron hojas fascículares, excepto algunas plantas de la familia 066, que por cierto presentó la menor tasa de formación de acículas.

De acuerdo con los resultados obtenidos, se observó una reducción general en el tamaño de las hojas primarias hacia finales del experimento, de tal forma que a las 30 semanas después del trasplante no se presentaron diferencias estadísticas entre familias, lo anterior fue debido a que cuando la planta empezó a desarrollar el epicotilo la longitud de hojas primarias fue de menor tamaño. 
Cuadro 3. Características morfológicas de plántulas de cuatro familias de Pinus montezumae Lamb., cultivadas en hidroponia.

\begin{tabular}{|c|c|c|c|c|}
\hline \multirow[t]{2}{*}{ Variable } & \multirow[t]{2}{*}{ Familia } & \multicolumn{3}{|c|}{ Semanas después del trasplante } \\
\hline & & 20 & 25 & 30 \\
\hline \multirow{4}{*}{$\begin{array}{l}\text { Diámetro del } \\
\text { hipocotilo } \\
(\mathrm{mm})\end{array}$} & 056 & $4.10 \mathbf{a}$ & $7.37 \mathbf{a}$ & $9.92 \mathbf{a}$ \\
\hline & 066 & $3.34 \mathbf{a}$ & $5.56 \mathrm{c}$ & $6.50 \mathrm{c}$ \\
\hline & 083 & $3.33 \mathbf{a}$ & $6.27 \mathbf{b}$ & $8.32 \mathbf{b}$ \\
\hline & 074 & $3.80 \mathrm{a}$ & $5.95 \mathbf{b}$ & $7.75 \mathbf{b}$ \\
\hline $\operatorname{DMS}_{(\alpha=0.05)}$ & & 0.97 & 0.66 & 1.09 \\
\hline Número de & 056 & $118.89 \mathbf{a}$ & $165.38 \mathbf{a}$ & $203.75 \mathbf{a}$ \\
\hline \multirow[t]{3}{*}{ hojas primarias } & 066 & $71.14 \mathbf{b}$ & $86.63 \mathrm{~b}$ & $152.50 \mathbf{b}$ \\
\hline & 083 & $106.68 \mathbf{a}$ & $155.75 \mathbf{a}$ & $183.12 \mathbf{a b}$ \\
\hline & 074 & $105.91 \mathbf{a}$ & $142.38 \mathbf{a}$ & $207.50 \mathbf{a}$ \\
\hline $\operatorname{DMS}_{(\alpha=0.05)}$ & & 14.82 & 24.48 & 46.07 \\
\hline \multirow{4}{*}{$\begin{array}{l}\text { Longitud del } \\
\text { epicotilo } \\
\text { (cm) }\end{array}$} & 056 & $0.96 \mathbf{a}$ & $6.07 \mathbf{a}$ & $15.06 \mathbf{a}$ \\
\hline & 066 & $0.35 \mathbf{a}$ & $0.97 \mathbf{b}$ & $4.75 \mathrm{~d}$ \\
\hline & 083 & $0.56 \mathbf{a}$ & $5.50 \mathbf{a}$ & $8.62 \mathrm{c}$ \\
\hline & 074 & $0.76 \mathbf{a}$ & $6.07 \mathbf{a}$ & $10.37 \mathbf{b}$ \\
\hline \multirow{5}{*}{$\begin{array}{l}\operatorname{DMS}_{(\alpha=0.05)} \\
\text { Longitud de } \\
\text { hojas primarias } \\
\quad(\mathrm{cm})\end{array}$} & & 0.66 & 1.09 & 1.58 \\
\hline & 056 & $16.69 \mathbf{a}$ & $15.50 \mathbf{a}$ & $13.13 \mathrm{a}$ \\
\hline & 066 & $11.31 \mathbf{b}$ & $11.75 \mathbf{b}$ & $12.25 \mathbf{a}$ \\
\hline & 083 & $13.27 \mathbf{b}$ & $13.94 \mathbf{a b}$ & $11.81 \mathbf{a}$ \\
\hline & 074 & $12.50 \mathrm{~b}$ & $14.25 \mathbf{a b}$ & $13.00 \mathbf{a}$ \\
\hline \multicolumn{2}{|l|}{$\operatorname{DMS}_{(\alpha=0.05)}$} & 2.83 & 2.68 & 1.37 \\
\hline \multirow{3}{*}{$\begin{array}{c}\text { Volumen de raíz } \\
\text { fresca } \\
\left(\mathrm{cm}^{3} \cdot \text { planta }^{-1}\right)\end{array}$} & 056 & $2.78 \mathbf{a}$ & $6.46 \mathbf{a}$ & $10.37 \mathbf{a}$ \\
\hline & 066 & $1.33 \mathbf{a}$ & $3.51 \mathrm{~b}$ & $6.30 \mathbf{b}$ \\
\hline & 083 & $1.78 \mathbf{a}$ & $5.39 \mathbf{a b}$ & $7.12 \mathbf{a b}$ \\
\hline \multirow[t]{2}{*}{$\operatorname{DMS}_{(\alpha=0.05)}$} & 074 & $1.41 \mathrm{a}$ & $5.11 \mathbf{a b}$ & $9.31 \mathbf{a b}$ \\
\hline & & 1.56 & 2.79 & 3.40 \\
\hline
\end{tabular}

Los valores seguidos por la misma letra, no difieren estadísticamente (Tukey, 0.05).

La familia 083 obtuvo el mayor número de hojas primarias, siendo significativamente superior a la familia 066 , las familias 056 y 074 presentaron un comportamiento intermedio entre las primeras familias mencionadas. Hubo diferencias significativas en la variable de volumen de raíz fresca. La familia que alcanzó el mayor VRF fue la 056, y la que obtuvo el menor fue la 66 , por su parte las familias 074 y 083 presentaron un comportamiento intermedio entre las dos primeras. 
A nivel de familias, pudo apreciarse en las variables morfológicas que se presentaron diferentes velocidades de crecimiento, y por lo tanto se supone diferente extracción nutrimental. Este comportamiento se le atribuyó a la expresión del carácter genotípico de cada familia, el cual inclusive varía de una planta a otra, aún siendo de la misma especie y cultivadas bajo un mismo tratamiento. Los cambios genotípicos entre especies, variedades, cultivares y familias obedecen a modificaciones en la anatomía, fisiología y metabolismo de la planta que se refleja en la absorción, transporte y utilización de los nutrimentos por las plantas (Marschner, 1995).

Es importante resaltar el efecto diferencial mostrado en el crecimiento del epicotilo entre familias como consecuencia del crecimiento vegetativo originado por una diferente constitución genética de las familias (Marschner, 1995). La familia 056 presentó un fenotipo superior en todos los aspectos a la familia 066, esto indicaría que aún en la especie típica (Pinus montezumae Lamb.) deben existir componentes y/o combinaciones génicas que controlan en crecimiento de las plántula. Una posible fundamentación a ésta aseveración es lo encontrado por Jasso (1990) en que la variedad Lindleyii de Pinus montezumae cuenta con un cierto porcentaje de plántulas con elongación de epicotilo en las primeras semanas después de la germinación y otro gran porcentaje del grupo (45\%) no.

\section{Producción de materia seca}

\section{Efecto de solución nutritiva}

La PMS de las plántulas de Pinus montezumae Lamb., cultivadas en las soluciones nutritivas utilizadas en la presente investigación (SNEST y SNCOO) muestra que hasta las 20 SDT no había diferencias estadísticas entre soluciones nutritivas en los diferentes órganos de las plantas (raíz, tallo y hojas; Cuadro 4).

Los efectos de solución nutritiva fueron notorios a las 25 SDT (semanas después del trasplante) para la materia seca foliar y total de las plantas y hasta las 30 SDT para la materia seca de la raíz; la materia seca del tallo, no presentó diferencias estadísticas durante el experimento. Independientemente de la solución nutritiva empleada, la acumulación de 
materia seca en los diferentes órganos de la planta a las 20 SDT fue mínima; los porcentajes de materia seca promedio fueron de $14.88,16.79$ y $22.19 \%$ respectivamente para raíz, tallo y hojas; en comparación con la materia seca total alcanzada por la planta al final del estudio (30 SDT). Lo que indica que tan sólo en un periodo de diez semanas la planta acumuló más del $80 \%$ de la materia seca obtenida al final del experimento. Esta rápida acumulación de materia seca en la planta durante las últimas diez semanas del estudio fue un indicador de máximo crecimiento y mayor demanda de nutrimentos, lo cual se comprobó con el efecto de solución nutritiva.

Cuadro 4. Producción de materia seca (g.planta ${ }^{-1}$ ) de plántulas de Pinus montezumae Lamb., cultivadas en hidroponia.

\begin{tabular}{|c|c|c|c|c|}
\hline \multirow[b]{2}{*}{ Órgano } & \multirow{2}{*}{$\begin{array}{r}\text { Solución } \\
\text { Nutritiva }\end{array}$} & \multicolumn{3}{|c|}{ Semanas después del trasplante } \\
\hline & & 20 & 25 & 30 \\
\hline \multirow[t]{2}{*}{ Raíz } & $\mathrm{SNCOO}$ & $0.230 \mathbf{a}$ & $0.869 \mathbf{a}$ & $1.703 \mathbf{a}$ \\
\hline & SNEST & $0.235 \mathbf{a}$ & $0.744 \mathbf{a}$ & $1.421 \mathrm{~b}$ \\
\hline $\operatorname{DMS}_{(\alpha=0.05)}$ & & 0.088 & 0.190 & 0.247 \\
\hline \multirow[t]{2}{*}{ Tallo } & SNCOO & $0.161 \mathbf{a}$ & $0.361 \mathbf{a}$ & $0.869 \mathbf{a}$ \\
\hline & SNEST & $0.108 \mathbf{a}$ & $0.297 \mathbf{a}$ & $0.733 \mathbf{a}$ \\
\hline$\left.{ }_{(\alpha M S}=0.05\right)$ & & 0.075 & 0.129 & 0.176 \\
\hline \multirow[t]{2}{*}{ Acículas (hojas) } & SNCOO & $0.690 \mathbf{a}$ & $1.980 \mathbf{a}$ & $3.685 \mathbf{a}$ \\
\hline & SNEST & $0.715 \mathbf{a}$ & $1.543 \mathrm{~b}$ & $2.646 \mathbf{b}$ \\
\hline $\operatorname{DMS}_{(\alpha=0.05)}$ & & 0.213 & 0.391 & 0.696 \\
\hline \multirow[t]{2}{*}{ Peso seco total } & SNCOO & $1.081 \mathbf{a}$ & $3.211 \mathbf{a}$ & $6.534 \mathbf{a}$ \\
\hline & SNEST & $1.058 \mathbf{a}$ & $2.615 \mathbf{b}$ & $5.085 \mathbf{b}$ \\
\hline $\operatorname{DMS}_{(\alpha=0.05)}$ & & 0.289 & 0.594 & 0.815 \\
\hline
\end{tabular}

Los valores seguidos por la misma letra, no difieren estadísticamente (Tukey, 0.05).

La materia seca producida por las plántulas de Pinus montezumae Lamb., abastecidas con la SNCOO en general superó a la materia seca de las plántulas abastecidas mediante la SNEST, lo cual estuvo asociado con un abastecimiento nutrimental diferente. La SNCOO en su formulación contiene $33.33 \%$ más nitrógeno, $50 \%$ más fósforo y $33.33 \%$ más potasio 
que la SNEST. Por lo tanto, se supone que las plántulas abastecidas con la SNCOO, tuvieron una mayor absorción y acumulación en el tejido vegetal de estos nutrimentos, durante el periodo de mayor crecimiento de la planta y de ahí que hayan crecido más y acumulado más materia seca que las plantas abastecidas con la SNEST.

Además de la concentración, la mayor absorción nutrimental pudo haber estado

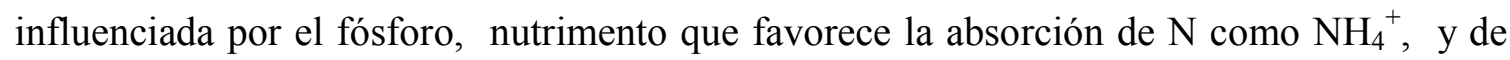
otros nutrimentos al incrementar el volumen radical.

De lo anterior se concluye que la materia seca acumulada por las raíces, que fue significativamente superior en las plantas abastecidas con la SNCOO, esté estrechamente relacionada con un mayor abastecimiento principalmente de $\mathrm{N}$ y $\mathrm{P}$ en esta solución, en comparación con la SNEST.

El tallo (hipocotilo + epicotilo) no presentó diferencias estadísticas, en la materia seca acumulada, aunque la SNCOO fue ligeramente superior a la SNEST. Este no fue un buen indicador del crecimiento del epicotilo, ya que esta variable si presentó diferencias estadísticas en su crecimiento en longitud. Lo anterior posiblemente estuvo relacionado con una mayor suculencia del tallo en las plantas abastecidas con la SNCOO, que por cierto contaron con un menor abastecimiento de calcio (170 ppm), en comparación con la SNEST (210 ppm). Por lo tanto una mayor longitud de epicotilo, no necesariamente significó una mayor cantidad de materia seca del tallo.

La materia seca foliar contribuyó de forma significativa para que la parte aérea de la planta presentara mayor cantidad de materia seca al no haber diferencias estadísticas en el tallo de la planta. Por lo que se supone que la mayor acumulación de nutrimentos estuvo en las hojas de las plantas. 


\section{Efecto de familia}

La producción en materia seca acumulada por planta correspondiente a cada familia, en los diferentes periodos de crecimiento y separada en cada uno de sus componentes (raíz, tallo y hojas) se presenta en el Cuadro 5. Es claro, la existencia de diferencias estadísticamente significativas, en las diferentes etapas de crecimiento entre familias.

Cuadro 5. Producción de materia seca (g.planta $\left.{ }^{-1}\right)$ de plántulas de cuatro familias de Pinus montezumae Lamb., cultivadas en hidroponia.

\begin{tabular}{|c|c|c|c|c|}
\hline \multirow[t]{2}{*}{ Parte de la planta } & \multirow[t]{2}{*}{ Familia } & \multicolumn{3}{|c|}{ Semanas después del trasplante } \\
\hline & & 20 & 25 & 30 \\
\hline \multirow[t]{4}{*}{ Raíz } & 056 & $0.325 \mathbf{a}^{4}$ & $1.005 \mathbf{a}$ & $1.894 \mathbf{a}$ \\
\hline & 066 & $0.189 \mathbf{a}$ & $0.526 \mathbf{b}$ & $1.182 \mathrm{c}$ \\
\hline & 074 & $0.237 \mathbf{a}$ & $0.954 \mathbf{a}$ & 1.399 bc \\
\hline & 084 & $0.174 \mathbf{a}$ & $0.801 \mathbf{a b}$ & $1.773 \mathbf{a b}$ \\
\hline \multicolumn{2}{|l|}{$\operatorname{DMS}_{(\alpha=0.05)}$} & 0.172 & 0.360 & 0.468 \\
\hline Tallo & 056 & $0.176 \mathbf{a}$ & $0.417 \mathbf{a}$ & $1.110 \mathbf{a}$ \\
\hline (hipocotilo & 066 & $0.073 \mathbf{a}$ & $0.199 \mathbf{a}$ & $0.431 \mathbf{c}$ \\
\hline \multirow[t]{3}{*}{ epicotilo) } & 074 & $0.148 \mathbf{a}$ & $0.376 \mathbf{a}$ & $0.761 \mathbf{b c}$ \\
\hline & 084 & $0.129 \mathbf{a}$ & $0.324 \mathbf{a}$ & $0.901 \mathbf{a b}$ \\
\hline & & 0.147 & 0.246 & 0.332 \\
\hline \multicolumn{5}{|l|}{$\operatorname{DMS}_{(\alpha=0.05)}$} \\
\hline \multirow[t]{4}{*}{ Acículas (Hojas) } & 056 & $0.992 \mathbf{a}$ & $2.343 \mathbf{a}$ & $3.454 \mathbf{a}$ \\
\hline & 066 & $0.460 \mathrm{~b}$ & $1.697 \mathbf{b}$ & $2.661 \mathbf{a}$ \\
\hline & 074 & $0.667 \mathbf{a}$ & $1.757 \mathbf{a b}$ & $3.118 \mathbf{a}$ \\
\hline & 084 & $0.704 \mathbf{a}$ & $1.251 \mathbf{b}$ & $3.428 \mathbf{a}$ \\
\hline $\operatorname{DMS}_{(\alpha=0.05)}$ & & 0.416 & 0.741 & 1.316 \\
\hline
\end{tabular}

" Los valores seguidos por la misma letra, no difieren estadísticamente (Tukey, 0.05).

La familia 056 se caracterizó por formar una mayor cantidad de raíz, con respecto a las familias 074,083 y 066 en los periodos correspondientes a las 20,25 y 30 SDT. A las 30 
SDT la materia seca en raíz para la familia 056 fue superior en un $60 \%$ a la familia 066 , y en un 26\%, y 6\% a las familias 074 y 083 ; respectivamente. Lo anterior se atribuyó a que las plantas que tuvieron una mayor cantidad de raíces se desarrollaron mejor en su parte aérea; lo que explica que la familia 066 haya resultado significativamente inferior en materia seca acumulada en tallos y hojas a las familias 056,074 y 083.

La materia seca acumulada por el tallo durante las 20 y 25 semanas después del trasplante fue ligeramente mas alta en la familia 056 (aunque sin ser significativamente diferente) con respecto las familias 066,074 y 083.

Al final del experimento (30 SDT) fue cuando realmente se observaron diferencias significativas entre familias. El mayor peso seco del tallo en las familias 056, 074 y 083 estuvo estrechamente relacionado con la mayor longitud del epicotilo alcanzado por las plántulas de Pinus montezumae Lamb., de estas familias, en comparación con la familia 066.

A las 20 SDT la materia seca foliar acumulada por las familias 056,074 y 083 no presentó diferencias estadísticas entre estas, pero si con la familia 066 que fue significativamente inferior a las primeras. Las hojas de las plantas de la familia 056 acumularon un $38.07 \mathrm{y}$ $29.80 \%$ mas materia seca que la familia 066 durante las 25 y 30 SDT; respectivamente.

En este estudio se obtuvo un crecimiento diferencial del epicotilo de plántulas de Pinus montezumae Lamb., entre las soluciones nutritivas y familias evaluadas. De acuerdo con los resultados obtenidos, las plántulas no entraron en la etapa de desarrollo característico de "estado cespitoso" por lo que no se puede afirmar que en este estudio se rompió el estado cespitoso. El crecimiento obtenido por el epicotilo independientemente de la solución nutritiva de las familias se atribuye a que las plántulas fueron cultivadas en un sistema hidropónico. Resultados similares en cuanto a respuesta al crecimiento de plántulas forestales nutridas con diferentes soluciones nutritivas han sido reportadas por Ingestad (1982); Landis, et al., (1989); López (1990); Travers (1965). 
Respecto a la respuesta observada entre familias, la superioridad de la familia 056 en todas las variables evaluadas pudo haber estado relacionada con su origen, ya que la semilla de ésta familia fue colectada en el área de certificación del área semillera de San Juan Tetla, Puebla. Por otra parte las familias 074 y 083 fueron obtenidas de colectas hechas en la franja de protección y la familia 066 fuera del área. El comportamiento diferente entre familias de plántulas de Pinus montezumae Lamb., es atribuido al genotipo diferente de cada familia. Al respecto, Marschner (1995), menciona que entre genotipos existen diferencias en las tasas de crecimiento, fenómeno que está determinado por una diferente morfología y fisiología de la planta, que se traduce en diferencias en el sistema radical, lo cual origina una diferente absorción nutrimental ante condiciones similares de abastecimiento de nutrimentos, lo que al final de cuentas se observa en un crecimiento vegetativo diferente en velocidad y desarrollo.

\section{CONCLUSIONES}

De los resultados obtenidos en la presente investigación se derivan las siguientes conclusiones: la velocidad de crecimiento de las plántulas de Pinus montezumae Lamb. fue diferente entre soluciones nutritivas, lo cual originó diferencias significativas en el crecimiento acumulado (en longitud y biomasa) de las variables medidas, en la etapa de máximo crecimiento de las plantas. La solución nutritiva Cooper produjo plántulas con mayores dimensiones en: diámetro del hipocotilo, longitud del epicotilo y materia seca. Independientemente de la solución nutritiva utilizada, en la presente investigación se estimuló el crecimiento del epicotilo, de Pinus montezumae Lamb. en un periodo de tiempo menor al que se presenta en forma natural cultivando las plantas en hidroponia. Entre familias se presentó un efecto diferencial en la velocidad de crecimiento de las plántulas de Pinus montezumae Lamb. La familia 056 fue la que obtuvo significativamente mayores crecimientos acumulados en diámetro del hipocotilo, longitud del epicotilo y materia seca por planta, y la familia 066 presentó los menores crecimientos; por otra parte las familias 074 y 083 tuvieron un comportamiento intermedio entre las familias 056 y 066. 


\section{LITERURA CITADA}

Alcántar, G. G. 1992. Análisis de agua y soluciones. In: G.J.D. Etchevers B. y A. Aguilar S (eds). Los análisis físicos y químicos, su aplicación en agronomía. Centro de Edafología, Colegio de Postgraduados, Montecillo, México. pp. 62-67.

Becerra, L. F. 1990. Problems of montezumae pine seedling establishment in central México: The need for renewing the pine forest. Tree News 8:5-7.

Calderón, P. N. 1995. Efecto de la salinidad en el crecimiento y absorción nutrimental de plantas micropropagadas de nopal (Opuntia spp.). Tesis de Licenciatura. Universidad Autónoma Chapingo. División de Ciencias Forestales. Chapingo México. 94 p.

Cortés, T. H. G. 1987. Análisis de la distribución estadística de las intensidades de lluvia en el area de estudio del CREZAS-CP. Tesis Profesional. UACh. Departamento de irrigación. Chapingo, Méx. 128 p.

Eguiluz, P. T. 1988. Distribución natural de los pinos en México. México, Centro de Genética Forestal, A.C. Nota Técnica No. 1. 6 p.

Furlani, P. R. 1995. Cultivo de alface pela técnica de hidroponía-NFT. Campinas. Instituto agronómico. Documentos IAC, 55: 18 p.

Hare, R. C. 1984. Stimulation of early height growth in longleaf pine with regulators. Can. J. For. Res. 14:459-462.

Hinsley, L. E. 1980. Fall fertilization helps longleaf pine nursery stock. Southern J. Appl. For. 4(3): 132-135.

Hughes, R. H., E. J. Jackson y H. R. Hart. 1971. Fertilization of young longleaf pine in a cultivated plantation. USDA. For. Serv. Southeast. For. Exp. Sta. Pap. SE-75. 8 p.

Ingestad, T. 1982. Relative addition rate and external concentration:driving variables used in plant nutrition research. Plant, Cell and Environment. 5:443-453.

Jasso, M. J. 1982. Ensayo preliminar de selección y cruzamientos en una población natural de Pinus montezumae Lamb. Tesis Profesional. U.A.Ch. Chapingo, Méx. México. 109 p. 
Jasso, M. J. 1990. Genetic Variation of Provenances and Differently Treated Stands of Pinus monteazumae in Mexico. Ph. D. Dissertation. Yale University. New Haven, CT. U.S.A. 318 p.

Kossuth, S. V. 1981. Shoting the grass stage of lonleaf pine with plant growth regulators. For. Sci. 27:400-401.

Lewis, C. E, G.W. Monson y R.J. Bonyata. 1985. Pensacola bahiagrass can be used to improv the forage resource when regenerating sothern pines. Southern J. Appl. For. 9(4):254-259.

López, L. M. A. 1990. Estudio de nutrición de Pinus patula Schl. et. Cham. en sistema hidropónico. Tesis de Licenciatura. División de Ciencias Forestales. Universidad Autónoma Chapingo. Chapingo, Méx. 181 p.

Marschner, H. 1995. Mineral Nutrition of Higher Plants. Academic Press. London. 674 p.

Mengel K y E.A. Kirkby. 1987. Principles of plant nutrition. $4^{\text {th }}$ Edition. International Potash Institute. Bern, Switzerland. 687 p.

Mexal, J. G. y T. D. Landis. 1990. Target seedling concepts: height and diameter. In: Rose, R. S.J. Campbell, y T.D. Landis (eds). Target seedling Simposium: Proceedings, Combined Meeting of the Western Forest Nursery Associations. General Technical Report R. M-200. pp. 17-36.

Mirov, N. T. 1967. The genus Pinus. New York: The Ronald Press Company. 602 p.

Musálem, M. A. 1985. Efecto de la intensidad de la luz en la germinación, desarrollo y sobrevivencia de plántulas de Pinus montezumae Lamb. Rev. Dasonomía Mexicana. (3) 5:25-34.

SAS Institute Inc. 1985. SAS. User's Guide: Statistics. Versión 5. SAS Institute Inc. Cary, N.C. U.S.A. 956 p.

Thomson, B. E. 1984. Establishing a vigorous nursery crop: bed preparation, seed sowing, and early seedling growth. In: duryea M.L. and T.D. Landis eds. Forest Nursery Manual: Production of bareroot seedlings. Martinus Nihoff/Dr. W. Junk Publishers. pp. 41-49.

Travers, W. W. G. 1965. The mineral nutrition of Pinus radiata seedlings. N.Z. For. Res. Notes. No. 40. pp. 1-10. 
Van, D. D. R. 1982. Relationship between spacing and nitrogen fertilization of seedlings in the nursery, seedling size and outplanting performance. Can. J. For. Res. 12: 865-875.

Vela, G. L. y Hernández, S. R. 1968. Influencia de la luz solar directa sobre el crecimiento de plántulas de vivero de Pinus patula Schl. et. Cham., y Pinus montezumae Lamb. Bol. Téc. 22. INIF-SFF-SAG. México. 15 p.

Vera, C. J. A. G. 1986. Estudio de algunos factores que influyen en la producción de Pinus montezumae Lamb., en vivero. Tesis de Maestría en Ciencias. Colegio de Postgraduados. Montecillo, México. 141 p.

\section{Jesús Jasso Mata}

Doctorado en Ciencias Forestales por la Universidad de Yale. Maestría en Ciencias Forestales por la Universidad de Yale. Ingeniero Agrónomo Especialista en Bosques por la Universidad Autónoma Chapingo. Miembro del Sistema Nacional de InvestigadoresCONACYT-México.

\section{J. Jesús Vargas Hernández}

Doctorado en Ciencias Forestales por la Universidad Estatal de Oregon. Maestría en Ciencias Forestales por el Colegio de Posgraduados. Ingeniero Agrónomo Especialistas en Bosques por la Universidad Autónoma Chapingo. Miembro del Sistema Nacional de Investigadores-CONACYT-México. 